\title{
Validade da Citologia e da Biópsia Orientada pela Colposcopia no Diagnóstico do Carcinoma Cervical Pré-clínico
}

\author{
Validity of Cytology and Colposcopy - Guided Biopsy for the Diagnosis of Preclinical \\ Cervical Carcinoma
}

Aldo Franklin Ferreira Reis ${ }^{1}$, Ricardo José de Oliveira e Silva ${ }^{1}$,
Maria José de Camargo ${ }^{2}$, Célia Maria Guimaraes da Silveira ${ }^{1}$

\section{RESUM0}

Objetivo: avaliar a eficácia da citologia e da biópsia orientada pela colposcopia na discriminação entre o carcinoma invasor pré-clínico e as lesões intra-epiteliais.

Pacientes e Métodos: 441 pacientes submetidas a conização, histerectomia e operação de Wertheim-Meigs, no periodo de 1978 a 1995, no Hospital Universitário Clementino Fraga Filho, Universidade Federal do Rio de Janeiro, Setor de Patologia Cervical. Foram efetuados os cálculos da sensibilidade, especificidade, valores preditivos, razão de verossimilhança e intervalos de confiança de cada exame, divididos em quatro categorias: normal e inflamatório; displasia leve e displasia moderada; displasia acentuada e carcinoma in situ, e carcinoma microinvasor e invasor. As biópsias foram analisadas como um todo e separadas pelo tipo de colposcopia (satisfatória e insatisfatória).

Resultados: a citologia mostrou sensibilidade de 50\%, especificidade de 89\%, valor preditivo positivo de $63 \%$ e valor preditivo negativo de $82 \%$. As razões de verossimilhança foram 4,4 para o diagnóstico de invasão, 0,7 para displasia acentuada e carcinoma in situ, 0,1 para displasia leve e moderada, 2,2 para normal e inflamatório e 0,6 para o conjunto de resultados negativos para invasão. A biópsia orientada pela colposcopia apresentou sensibilidade de $50 \%$, especificidade de $100 \%$, valor preditivo positivo de $100 \%$ e valor preditivo negativo de 83\%. As razões de verossimilhança foram: tendendo ao $\infty$ para o resultado de invasão, 0,5 para displasia acentuada e carcinoma in situ, 0,2 para displasia leve e moderada, 0,3 para normal e inflamatório e 0,5 para o conjunto de resultados negativos para invasão. A biópsia orientada pela colposcopia satisfatória com lesão visivel mostrou sensibilidade de 59\%, especificidade de $100 \%$, valor preditivo positivo de $100 \%$ e valor preditivo negativo de $83 \%$. As razões de verossimilhança foram: tendendo ao $\infty$ para o resultado positivo de invasão, 0,5 para displasia acentuada e carcinoma in situ, zero para displasia leve e moderada, zero para negativo e inflamatório e 0,4 para o conjunto de resultados negativos para invasão.

PALAVRAS-CHAVE: Colo do útero: câncer. Colo do útero: lesões pré-neoplásicas. Colpocitologia. Colposcopia.

\section{Introdução}

\footnotetext{
${ }^{1}$ Hospital Universitário Clementino Fraga Filho da Universidade Federal do Rio de Janeiro

${ }^{2}$ Instituto Fernandes Figueira da Fundação Instituto Oswaldo Cruz.

Correspondencia:

Aldo Franklin Ferreira Reis

Rua Maia Lacerda 637/702 - Rio Comprido

20250-001 - Rio de Janeiro - RJ
}

Os tumores do colo uterino são os mais freqüentes entre as mulheres brasileiras, sendo responsáveis por $15 \%$ de todos os tumores malignos. Nas regiões menos desenvolvidas do País, 
são causa de cerca de $20 \%$ dos óbitos por neoplasias e cidades como Recife, Fortaleza, Belém e Goiânia apresentam as maiores incidências descritas na literatura mundial. As elevadas taxas de diagnóstico do carcinoma invasor indicam a baixa cobertura populacional do rastreamento colpocitológico e dão indícios de alto grau de evasão do seguimento nos casos rastreados.

O correto diagnóstico das lesões previamente rastreadas pela citologia é um aspecto crucial para a conduta subseqüente. Quando um grande número de pacientes é avaliado com citologia cervical atípica, verifica-se que um terço destas mulheres têm lesões atribuídas a infecções ou atrofia. Nos dois terços restantes, observamos lesões neoplásicas, sendo que em um percentual pequeno haverá o diagnóstico definitivo de carcinoma microinvasor ou invasor.

Quanto maior a cobertura pelo rastreamento citológico, maior o número de pacientes que necessitarão de diagnóstico preciso e ulterior conduta terapêutica. A invasão do estroma representa importante alteração qualitativa e faz jus à especial atenção no seu diagnóstico. Cada caso de câncer invasor não-identificado pelos métodos diagnósticos pós-rastreamento resulta em retardo no tratamento adequado e piora no prognóstico.

Os métodos utilizados no diagnóstico e tratamento por vezes se confundem, não havendo consenso em relação ao que deva ser considerado diagnóstico suficiente para que se estabeleça o tratamento. Nas últimas décadas difundiu-se a utilização de métodos destrutivos quando a biópsia orientada pela colposcopia constatava lesão nãoinvasora ${ }^{10,22}$. Esta abordagem vem sendo questionada em função da necessidade de maior amostra de tecido para exame e diagnóstico definitivo ${ }^{2,11,15,17,22}$.

A boa prática da colposcopia exige treinamento adequado e cuidadosa correlação entre os achados visuais e a histologia ${ }^{1,12,21}$. Sua indicação primordial é a localização e delimitação das lesões epiteliais. Identificada a lesão suspeita é, então, realizada a biópsia, que de maneira indireta mede a eficácia da colposcopia. A colposcopia é, supostamente, o meio de "explicar" o que foi detectado pela citologia. Como os colposcopistas sempre tomam conhecimento do diagnóstico citológico, naturalmente dedicam maior atenção aos casos cujos laudos descrevem alterações mais intensas ${ }^{24}$. A maioria dos autores acredita que todas as lesões suspeitas devam ser biopsiadas e sustentam a realização de mais de uma biópsia ${ }^{6,8,10}$. Como os sinais colposcópicos são indiretos e inespecíficos, a restrição do número de biópsias propicia o erro diagnóstico. Por outro lado, aumentando o número de biópsias elevam-se também os custos.

Podemos citar como fatores que influenciam a validade da colposcopia: o tamanho e a gravidade da lesão, o número de biópsias, a idade das pacientes, a variabilidade na observação, a variabilidade da interpretação histológica, a inflamação e o diagnóstico citológico. O tamanho da lesão está relacionado à gravidade do resultado histológico. A associação entre tamanho e eficácia diagnóstica é inversa; as lesões focais são de identificação mais fácil do que as lesões dispersas sobre dois ou mais quadrantes cervicais. É baixa a freqüência de lesões diminutas em pacientes com neoplasia invasora ${ }^{24}$. Existe associação entre a idade da paciente e a gravidade da lesão no continuum da doença cervical. Nas idosas, o diagnóstico é dificultado pela localização da junção escamocolunar, que tende a migrar para o canal cervical, com conseqüente necessidade de aumento no número de indicações da conização ${ }^{24}$.

Há grande variabilidade na interpretação histopatológica. Ismail et al. ${ }^{7}$ estudaram, entre oito histopatologistas, a variabilidade em diagnosticar e graduar neoplasia intra-epitelial cervical em 100 biópsias colposcopicamente dirigidas. A concordância entre os observadores foi excelente nas lesões invasoras, moderadamente boa para as displasias acentuadas e carcinoma in situ, e pobre para as leves e moderadas. Todavia, os casos com carcinoma invasor constituíram menos de $2 \%$ da amostra, não permitindo a análise da discordância quanto a lesão mais graves. Há, de fato, dificuldade em distinguir as lesões intra-epiteliais dos estágios iniciais de invasão $0^{3,14}$. Os processos inflamatórios do colo e vagina podem confundir os colposcopistas simulando o pontilhado. Helmerhorst et al. ${ }^{6}$ encontraram queda da eficácia das biópsias colposcópicas na presença de colpite por Gardnerella vaginalis.

A validade da biópsia orientada pela colposcopia foi estudada por diversos autores ${ }^{4,8,9,14,16,21,23}$. Stafl e Mattingly ${ }^{21}$ compararam o diagnóstico da biópsia orientada com o exame do cone em 217 pacientes. A sensibilidade para o diagnóstico de invasão (calculada por nós) foi de $71 \%$. Contudo, eram apenas 17 casos de carcinoma invasor. Donohue e Meriwether ${ }^{4}$, Helmerhorst et al. ${ }^{6}$, Kirkup e Hill ${ }^{9}$, Ronk et al. ${ }^{14}$, Veridiano et al. ${ }^{23}$ e Wetrich ${ }^{24}$ utilizaram metodologia de concordância dos diagnósticos da biópsia e do cone e ainda contavam com pequeno número de casos de carcinoma invasor. 
O dilema é, no entanto, saber a eficácia do método para distinguir as lesões limitadas ao epitélio daquelas que iniciam a invasão estromal. Benedet et al. $^{3}$ estudaram 180 pacientes com microcarcinoma e carcinoma oculto, criticando as conclusões dos trabalhos que pretenderam avaliar a validade da biópsia para diferenciar a neoplasia intra-epitelial da invasão inicial sem contar com número significativo de casos nas quais a invasão ocorria ou sem utilizar um adequado padrão de referência.

O objetivo deste estudo foi avaliar a eficácia da citologia e da biópsia orientada pela colposcopia, na discriminação entre a lesão intra-epitelial e o carcinoma cervical invasor pré-clínico.

\section{Pacientes e Métodos}

Foram analisadas 441 pacientes submetidas a conização (354), operação de Wertheim-Meigs (83) e histerectomia total (4), no Serviço de Ginecologia do Hospital Universitário Clementino Fraga Filho da Universidade Federal do Rio de Janeiro, no período de 1978 a 1995. Foram excluídas as pacientes que apresentavam evidência clínica de carcinoma invasor. Quando a propedêutica por meio da citologia, colposcopia e biópsia não permitia excluir a presença de lesão invasora, a conização foi utilizada com este objetivo.

O mais grave dentre os diagnósticos histológicos (biópsia, cone, útero) foi considerado como diagnóstico definitivo. Foi definido como diagnóstico citológico e diagnóstico da biópsia o resultado mais grave da mesma paciente. A colposcopia foi subdividida em satisfatória e insatisfatória e, também, por apresentar ou não lesão visivel.

A análise dos dados foi realizada comparando-se com o diagnóstico definitivo o resultado da citologia e o resultado da biópsia orientada pela colposcopia. A análise foi feita globalmente e pela seleção daquelas cujas colposcopias identificaram lesão suspeita. Foram calculadas a sensibilidade, a especificidade, o valor preditivo positivo, o valor preditivo negativo e a razão de verossimilhança para o diagnóstico de carcinoma microinvasor e invasor pré-clínico em relação à lesão intra-epitelial. Para efeito de cálculo, os resultados dos exames foram subdivididos em quatro grupos: normal e inflamatório, displasia leve e moderada, displasia acentuada e carcinoma in situ e carcinoma microinvasor e invasor. Esta subdivisão obedeceu a critérios clínicos e prognósticos, objetivando a obtenção de número adequado de casos para análise em cada categoria.

\section{Resultados}

A Tabela 1 mostra a relação entre o resultado da citologia e o diagnóstico definitivo. Encontramos sensibilidade de $50 \%$, especificidade de $89 \%$, valor preditivo positivo de $63 \%$ e valor preditivo negativo de $82 \%$. A razão de verossimilhança para invasão foi de 4,4 para o resultado de invasão, de 0,7 para displasia acentuada e carcinoma in situ, 0,1 para displasia leve e moderada, 2,2 para citologias negativas ou inflamatórias e 0,6 para o conjunto de resultados negativos para invasão (Tabela 2).

Tabela 1 - Comparação entre o diagnóstico citológico e o diagnóstico definitivo (histopatologia).

\begin{tabular}{lccr}
\hline \multirow{2}{*}{ Citologia } & \multicolumn{2}{c}{ Diagnóstico definitivo } & \\
& Invasor & Não-invasor & Total \\
\hline Invasor & 62 & 36 & 98 \\
Displasia acentuada e carcinoma in situ & 52 & 189 & 241 \\
Displasia leve e displasia moderada & 4 & 85 & 89 \\
Normal e inflamatório & 6 & 7 & 13 \\
Total & 124 & 317 & 441 \\
\hline
\end{tabular}

Sensibilidade $=50 \%$

Especificidade $=89 \%$

Valor preditivo positivo $=63 \%$

Valor preditivo negativo $=82 \%$

Intervalo de confiança a $95 \%$

$40,96-59,04$

$84,51-91,81$

$62,74-63,66$

$77,85-85,95$

Tabela 2 - Razões de verossimilhança para a citologia

\begin{tabular}{lcc}
\hline Citologia & $\begin{array}{c}\text { Razão de } \\
\text { verossimilhança }\end{array}$ & $\begin{array}{c}\text { Intervalo de } \\
\text { confiança a 95\% }\end{array}$ \\
\hline Invasor & 4,4 & $3,09-6,27$ \\
Não-invasor & 0,56 & $0,46-0,66$ \\
Displasia acentuada e carcinoma in situ & 0,70 & $0,55-0,87$ \\
Displasia leve e displasia moderada & 0,12 & $0,04-0,30$ \\
Normal e inflamatório & 2,19 & $0,78-6,08$
\end{tabular}

A Tabela 3 mostra o estudo das biópsias orientadas pela colposcopia comparado com o diagnóstico definitivo de invasão. A sensibilidade foi de $50 \%$ e a especificidade $100 \%$. O valor preditivo positivo foi de $100 \%$ e o valor preditivo negativo $83 \%$. As razões de verossimilhança foram 0,3 para os resultados negativo e inflamatório, 0,2 para displasia leve e moderada, 0,5 para displasia acentuada e carcinoma in situ, tendendo ao $\infty$ para o resultado positivo de invasão e 0,5 para o conjunto de 
resultados negativos para invasão (Tabela 4).

Tabela 3 - Comparação entre o resultado da biópsia e o diagnóstico definitivo (cirúrgico).

\begin{tabular}{lccr}
\hline \multirow{2}{*}{ Biópsia } & \multicolumn{3}{c}{ Diagnóstico definitivo } \\
& Invasor & Não-invasor & Total \\
\hline Invasor & 56 & 0 & 56 \\
Displasia acentuada e carcinoma in situ & 50 & 205 & 255 \\
Displasia leve e displasia moderada & 4 & 46 & 50 \\
Normal e inflamatório & 3 & 28 & 31 \\
Total & 113 & 279 & 392 \\
\hline
\end{tabular}

Sensibilidade $=50 \%$

Especificidade $=100 \%$

Valor preditivo positivo $=100 \%$

Valor preditivo negativo $=83 \%$

Intervalo de confiança a $95 \%$

$40,10-59,04$

$78,60-87,40$

Tabela 4 - Razões de verossimilhança para a biópsia sob colposcopia

\begin{tabular}{lcc}
\hline Biópsia & $\begin{array}{c}\text { Razão de } \\
\text { verossimilhança }\end{array}$ & $\begin{array}{c}\text { Intervalo de } \\
\text { confiança a 95\% }\end{array}$ \\
\hline Invasor & $\infty$ & - \\
Não-invasor & 0,50 & $0,41-0,59$ \\
Displasia acentuada e carcinoma in situ & 0,54 & $0,43-0,66$ \\
Displasia leve e displasia moderada & 0,21 & $0,08-0,54$ \\
Normal e inflamatório & 0,26 & $0,86-0,79$ \\
\hline
\end{tabular}

Na Tabela 5 comparamos o resultado da biópsia efetuada durante colposcopia satisfatória com lesão visível com o diagnóstico definitivo de invasão. A sensibilidade foi de 59\%, especificidade $100 \%$, o valor preditivo positivo foi de $100 \%$ e o valor preditivo negativo $83 \%$. As razões de verossimilhança foram de zero para os resultados negativo e inflamatório, zero para displasia leve e moderada, 0,5 para displasia acentuada e carcinoma in situ, tendendo ao $\infty$ para o resultado positivo de invasão e 0,4 para o conjunto dos resultados negativos para invasão (Tabela 6).

Tabela 5 - Comparação entre o resultado da biópsia orientada por colposcopia satisfatória com lesão visível e o diagnóstico definitivo.

\begin{tabular}{lccr}
\hline \multirow{2}{*}{ Biópsia } & \multicolumn{2}{c}{ Diagnóstico definitivo } & \\
& Invasor & Não-invasor & Total \\
\hline Invasor & 39 & 0 & 39 \\
Displasia acentuada e carcinoma in situ & 27 & 111 & 138 \\
Displasia leve e displasia moderada & 0 & 15 & 15 \\
Normal e inflamatório & 0 & 4 & 4 \\
Total & 66 & 130 & 196 \\
\hline
\end{tabular}

Sensibilidade $=59 \%$

Intervalo de confiança a $95 \%$

Especificidade $=100 \%$

$46,34-70,78$

Valor preditivo positivo $=100 \%$

Valor preditivo negativo $=83 \%$

$76,32-89,20$
Tabela 6 - Razões de verossimilhança para a biópsia orientada por colposcopia satisfatória com lesão visivel.

\begin{tabular}{lcc}
\hline Biópsia & $\begin{array}{c}\text { Razão de } \\
\text { verossimilhança }\end{array}$ & $\begin{array}{c}\text { Intervalo de } \\
\text { confiança a 95\% }\end{array}$ \\
\hline Invasor & $\infty$ & \\
Não-invasor & 0,41 & $0,30-0,53$ \\
Displasia acentuada e carcinoma in situ & 0,4791 & $0,35-0,62$ \\
Displasia leve e displasia moderada & Zero & \\
Normal e inflamatório & Zero &
\end{tabular}

A Tabela 7 mostra a comparação da biópsia orientada por colposcopia insatisfatória com lesão visível com o diagnóstico definitivo de invasão. A sensibilidade foi de $39 \%$, a especificidade $100 \%$, o valor preditivo positivo foi de $100 \%$ e o valor preditivo negativo $83 \%$. As razões de verossimilhança foram 0,02 para os resultados normal e inflamatório, 0,15 para displasia leve e displasia moderada, 0,75 para displasia acentuada e carcinoma in situ, tendendo ao $\infty$ para invasor $\mathrm{e}$ de 0,6 para o conjunto dos diagnósticos negativos para invasão (Tabela 8).

Tabela 7 - Comparação entre os resultados da biópsia orientada por colposcopia insatisfatória com lesão visível e o diagnóstico definitivo

\begin{tabular}{lccrr}
\hline Biópsia & \multicolumn{2}{c}{ Diagnóstico definitivo } \\
\hline Invasor & 17 & 0 & 17 \\
Displasia acentuada e carcinoma in situ & 22 & 91 & 113 \\
Displasia leve e displasia moderada & 4 & 24 & 28 \\
Normal e inflamatório & 1 & 22 & 23 \\
Total & 44 & 137 & 181 \\
\hline
\end{tabular}

Sensibilidade $=39 \%$

Intervalo de confiança a $95 \%$

Especificidade $=100 \%$

Valor preditivo positivo $=100 \%$

Valor preditivo negativo $=83 \%$

$73,34-89,74$

Tabela 8 - Razões de verossimilhança para a biópsia orientada por colposcopia insatisfatória com lesão visível.

\begin{tabular}{lcc}
\hline Biópsia & $\begin{array}{c}\text { Razão de } \\
\text { verossimilhança }\end{array}$ & $\begin{array}{c}\text { Intervalo de } \\
\text { confiança a 95\% }\end{array}$ \\
\hline Invasor & $\infty$ & $0,47-0,74$ \\
Não-invasor & 0,61 & $0,53-1,00$ \\
Displasia acentuada e carcinoma in situ & 0,75 & $0,06-0,40$ \\
Displasia leve e displasia moderada & 0,15 & $0,01-0,19$ \\
Normal e inflamatório & 0,02 & \\
\hline
\end{tabular}

\section{Discussão}

A validação de um teste diagnóstico deve ser feita pela comparação com um padrão de referência apropriado. Em se tratando de testes destinados a 
neoplasias epiteliais cervicais, o padrão de referência consagrado é a histopatologia realizada em peça cirúrgica suficientemente ampla ${ }^{3,13}$. A comparação de todas as pacientes submetidas ao teste com o padrão de referência constituiria um estudo ideal. Por motivos éticos não é possivel submeter à conização pacientes com biópsias negativas ou que apresentem lesões intra-epiteliais leves ou moderadas sem indicação cirúrgica. Embora baixa, a morbidade desta operação deve ser considerada. À conização pode ser atribuída a propriedade de diagnosticar de maneira definitiva as alterações epiteliais. Há, ainda, consenso sobre sua capacidade de tratá-las eficazmente.

Ao selecionarmos para o estudo pacientes que foram submetidas à cirurgia, observa-se como vício de seleção a maior representação de lesões mais graves. Por outro lado, desta forma foi possivel concentrar número significativo de lesões invasoras iniciais, objetivo de nossa análise.

Tomamos como referencial teórico a vasta experiência mundial no uso da colposcopia como método para localizar e delimitar a extensão das lesões. A biópsia orientada pela colposcopia, na verdade, reúne dois métodos propedêuticos. O primeiro, a colposcopia, apresenta grande subjetividade, sendo a variabilidade interobservadores de difícil verificação. A falha no reconhecimento de uma colposcopia insatisfatória e a não-identificação dos vasos atípicos constituem erros comuns na avaliação colposcópica. A biópsia, embora apresente considerável variabilidade interobservadores, permite revisão a qualquer tempo, à semelhança da citologia ${ }^{7}$. A validade da biópsia é influenciada por todos os aspectos interferentes na interpretação colposcópica.

A partir da década de 70 , com a aceitação da colposcopia na América do Norte e a supervalorização de sua validade na orientação da biópsia, difundiram-se os chamados métodos destrutios das lesões diagnosticadas como intraepiteliais ${ }^{10,16,23}$.

A popularização das diversas formas de tratamento dessas lesões trouxe como vantagens a redução dos custos e da morbidade inerentes à conização. Porém, relatos de carcinomas invasores em pacientes submetidas à terapêutica destrutiva ${ }^{2,11,15,17,22}$ conduziram a uma melhor avaliação da validade dos métodos diagnósticos. Percebeu-se que a afirmação de excelência da biópsia orientada pela colposcopia para diagnosticar as neoplasias cervicais foi precipitada. Benedet et $a .^{3}$ reuniram número significante de lesões invasoras e verificaram sensibilidade de $79 \%$ para a biópsia orientada pela colposcopia satisfatória, permitindo a análise adequada da validade do método para o diagnóstico diferencial entre lesões intra-epiteliais e invasoras pré-clinicas.

$E$ É freqüente na literatura a presença de estudos de verificação da eficácia da biópsia por meio da concordância do diagnóstico, comparando os diversos graus de lesões epiteliais com o padrão de referência ${ }^{4,6,9,14,23,24}$. A análise da concordância entre as pessoas comprovadamente portadoras da doença corresponde à sensibilidade. O diagnóstico discordante, entretanto, merece interpretação de sua importância clínica. Desta forma, o resultado do exame histológico do cone, ao revelar lesão menos grave do que a biópsia, não significa resultado falso-positivo da biópsia, mas a possibilidade já suficientemente demonstrada da erradicação da lesão mais grave naquele procedimento. No entanto, uma biópsia menos grave que o cone representa resultado falsonegativo. Esta é importante, mas a análise de concordância atribui o mesmo valor a qualquer discordância. Tais estudos, em geral, pouco informam sobre o tipo ou importância do erro diagnóstico e desconsideram a probabilidade da ocorrência de concordância ao acaso.

Entendemos que a análise que melhor reflete a problemática ora em estudo é aquela que valoriza os erros potencialmente mais danosos, especificamente o resultado falso-negativo para invasão.

Entre nós, a validade da biópsia orientada pela colposcopia também mereceu atenção. Simões et al. ${ }^{19}$ obtiveram três biópsias positivas para invasão em 31 casos de carcinoma invasor préclínico submetidos à conização. Concluíram que "em casos de diagnóstico de displasia acentuada e carcinoma in situ, a conização deverá estar obrigatoriamente situada entre a biópsia e o procedimento terapêutico que venha a ser adotado". Silva ${ }^{18}$, após excluir os casos nos quais a biópsia indicava invasão, submeteu à conização 133 pacientes com diagnóstico citológico de carcinoma in situ ou displasia acentuada e biópsia positiva para neoplasia intra-epitelial. Obteve como resultado histopatológico dos cones 16 carcinomas invasores. Nós associamos a citologia à biópsia orientada por colposcopia satisfatória com lesão visivel e verificamos sensibilidade de $76,2 \%$.

A análise baseada em longo período de observação está sujeita a modificações que ocorrem com o decorrer do tempo. Os conceitos e condutas são periodicamente atualizados, prejudicando a uniformidade nos protocolos de atendimento. Julgamos ainda importantes as mudanças de pessoal responsável pelo atendimento ambulatorial, 
uma vez que a colposcopia é método que depende de interpretação subjetiva. A partir de 1988 houve modificação da clientela atendida no Setor de Patologia Cervical com o aporte de pacientes referidas pelo PITEC (Programa Integrado Tecnológico em Citopatologia) com colpocitologia de rastreamento positiva, aumentando consideravelmente o número de neoplasias em nosso material.

A interpretação do desempenho de um teste no seu uso clínico implica o relacionamento de uma probabilidade pós-teste da doença, denotando o caráter probabilístico do processo diagnóstico. A prevalência pode ser considerada uma probabilidade pré-teste e o valor preditivo uma probabilidade pós-teste. Maior relevância tem sido atribuída ao uso da razão de verossimilhança por ser independente da prevalência, o que não ocorre com o valor preditivo. Apesar da aparente complexidade, verifica-se a cada dia sua maior aplicação na prática médica com a publicação de valores de razão de verossimilhança para diversos métodos propedêuticos. Entendemos que essa metodologia mais racional deva ser incorporada, de tal forma que o encadeamento das etapas do processo diagnóstico seja concluído ao atingir-se um limiar de decisão, que permita confirmar o diagnóstico ou descartar a possibilidade de doença.

$\mathrm{Na}$ interpretação dos dados desse estudo é necessário levar em consideração a prevalência de doença (no caso, carcinoma oculto) nos diversos ambientes em que atuamos. É necessário também interpretar os intervalos de confiança que quando muito grandes, indicam imprecisão do dado por tamanho amostral muito pequeno. Só devemos dar crédito aos dados que apresentam intervalos de confiança bem pequenos.

A citologia apresentou sensibilidade de 50\%, superior ao observado por Benedet et al. ${ }^{3}$. A especificidade de $89 \%$ implica $11 \%$ de resultados falso-positivos, indicando maior precisão diagnóstica nas lesões intra-epiteliais. O valor preditivo positivo de $63 \%$ deve ser interpretado com cautela, em face da alta prevalência de lesões invasoras: $28 \%$ em nosso material. Em outros ambientes dificilmente encontraremos tal prevalência, somente explicada pela seleção dos casos estudados. A razão de verossimilhança demonstrou pequena capacidade de confirmação do diagnóstico de invasão $(4,4)$, ou seja, pouco modifica a probabilidade pré-teste de doença. No entanto, a razão de verossimilhança para displasia leve/moderada $(0,1)$ modifica intensamente a probabilidade pré-teste (20\%).

Giard e Hermans ${ }^{5}$ calcularam as razões de verossimilhança para a colpocitologia, obtendo valores diferentes dos observados por nós. Além da utilização de padrão de referência diferente, a divergência pode ser imputada ao pequeno número de displasias acentuadas e carcinomas in situ (74), sendo ainda menor o número de lesões invasoras (15) nos 3.585 casos estudados. Surpreendeu-nos no nosso material a razão de verossimilhança mais alta para as citologias com resultado normal ou inflamatório $(2,2)$ do que para aquelas com resultado de displasia leve ou moderada $(0,1)$. Tal resultado aparentementemente ilógico deve ser atribuído ao pequeno número de casos nesse agrupamento (3\%) ou a incapacidade da citologia de discriminar as lesões mais sutis.

Em relação à biópsia, a sensibilidade foi de $50 \%$ para qualquer tipo de colposcopia. O valor preditivo positivo da biópsia é obviamente 100\%, ao passo que o valor preditivo negativo foi de $83 \%$; conseqüentemente, $17 \%$ das biópsias tiveram resultado falso. No ambiente de clinica de patologia cervical em que a prevalência de invasão é muito menor que a nossa, o valor preditivo negativo deve chegar perto de 100\%. Como dito anteriormente, este erro é muito grave para se correr este pequeno risco.

As razões de verossimilhança encontradas não geram grandes modificações nas probabilidades pré-teste de lesão invasora, exceção feita, é claro, ao resultado positivo de invasão. Nas biópsias em que a colposcopia foi satisfatória e a lesão visível, a sensibilidade atingiu $59 \%$ e o valor preditivo negativo manteve-se em $83 \%$. A razão de verossimilhança para o diagnóstico de displasia acentuada e carcinoma in situ $(0,5)$ pouco modifica a probabilidade pré-teste. Entretanto, para o diagnóstico de displasia leve ou moderada (zero), gera grande modificação. A exclusão de lesão invasora seria possivel, indicando tratamento conservador nestas circunstâncias. Na colposcopia insatisfatória com lesão visível a razão de verossimilhança acarreta queda pequena na probabilidade pré-teste, não excluindo a lesão invasora.

Nas duas últimas décadas, as evidências das limitações da biópsia ensejaram o desenvolvimento de métodos que pudessem cumprir o princípio da remoção sem prejuizo da capacidade diagnóstica. Aperfeiçoaram-se as alças diatérmicas. Variadas na forma, exigem ponderação sobre o estabelecimento dos limites de sua aplicabilidade. A utilização de alças diatérmicas pequenas (até 1,0 centímetro de diâmetro), destinadas à exérese de lesões periorificiais, constitui procedimento singelo, assemelhado em custo e morbidade às biópsias ${ }^{12}$. 
Conquanto a excisão da zona de transformação com alça diatérmica larga apresente menor morbidade e menores custos do que a conização convencional com bisturi ${ }^{12,20}$, sua utilização indiscriminada pode acarretar retrocesso ao período anterior ao uso da colposcopia na América do Norte. No estudo de Alvarez et al. ${ }^{1}$, $32,5 \%$ das pacientes submetidas à excisão com alça diatérmica não apresentavam qualquer evidência de neoplasia intra-epitelial e em $26,5 \%$ a displasia leve foi o diagnóstico final.

Estes dados não nos permitem realizar tratamento destrutivo sem que corramos algum risco de deixar passar um carcinoma incipiente. $\mathrm{Na}$ experiência individual não verificamos isto rapidamente, porque a incidência destas invasões é muito pequena, mas com o uso indiscriminado em massa os casos vão aparecendo ${ }^{2,11,15,17,22}$. De outro lado, não vemos com entusiasmo qualquer proposta que coloque a colposcopia e a biopsia orientada pela colposcopia em segundo plano ${ }^{1}$.

Queremos ressaltar a necessidade de utilização de metologia adequada na investigação de métodos diagnósticos em patologia cervical, para evitar prejuízos no tratamento de pacientes com lesão invasora inicial que ainda têm prognóstico razoável apesar da invasão do estroma.

\section{SUMMARY}

Purpose: to evaluate the efficacy of cytology and colposcopydirected biopsy to distinguish preclinical invasive cervical carcinoma from intraepithelial lesions.

Patients and Methods: 441 patients submitted to conization, hysterectomy and Wertheim-Meigs operation from 1978 to 1995 in the University Hospital "Clementino Fraga Filho", Federal University of Rio de Janeiro, Cervical Pathology Outpatient Clinic. We estimated sensitivity, specificity, predictive values, likelihood ratio and confidence intervals of each study, which were divided into four classes: 1) normal and inflammatory; 2) mild and moderate dysplasias; 3) severe dysplasia and carcinoma in situ; 4) microcarcinoma and invasive carcinoma. Biopsies were analyzed as a whole and separated in accordance with the type of the colposcopic result (satisfactory and unsatisfactory).

Results: cytology has shown sensitivity of $50 \%$, specificity of $89 \%$, positive predictive value of $63 \%$ and negative predictive value of $82 \%$. The likelihood ratios were 4.4 for stromal invasion diagnosis, 0.7 for severe dysplasia and carcinoma in situ, 0.1 for mild and moderate dysplasia, 2.2 for normal and inflammatory report and 0.6 for the negative results for invasion as a whole. Satisfactory colposcopic guided biopsy white a visible lesion showed sensitivity of $59 \%$, specificity of $100 \%$ positive predictive value of $100 \%$ and negative predictive value of $83 \%$. Likelyohood ratios were: tending to infinity for invasion, 0.5 for severe dysplasia and carcinoma in situ, zero for mild and moderate dysplasia, zero for negative and inflammatory and 0.4 for all negative results for invasion.

KEY WORDS: Cervix. Uterine cervix: neoplasms. Preneoplastic lesions. Vaginal citology. Colposcopy. validade da citologia e da biópsia orientada pela colposcopia no diagnóstico do carcinoma cervical pré-clínico

\section{Referências}

1. Alvarez RD, Helm CW, Edwards RP, Naumann RW, Partridge EE, Shingleton HM, et al. Prospective randomized trial of LLETZ versus laser ablation in patients with cervical intraepithelial neoplasia. Gynecol Oncol 1994; 52: 175-9.

2. Anderson MC. Invasive carcinoma of the cervix following local destructive treatment for cervical intraepithelial neoplasia. Br J Obstet Gynaecol 1993; 100: 657-63.

3. Benedet JL, Anderson GH, Boyes DA. Colposcopic accuracy in the diagnosis of microinvasive and occult invasive carcinoma of the cervix. Obstet Gynecol 1985; 65: 557-62.

4. Donohue LR, Meriwether W. Colposcopy as a diagnostic tool in the investigation of cervical neoplasia. Am J Obstet Gynecol 1972; 113: 10710.

5. Giard RW, Hermans J. The evaluation and interpretation of cervical cytology: application of the likelihood ratio concept. Cytopathology 1993; 4: 131-7.

6. Helmerhorst TJ, Dijkhuizen GH, Calame JJ, Kwikkel HJ, Stolk JG. The accuracy of colposcopically directed biopsy in the diagnosis of CIN. Eur J Obstet Gynecol Reprod Biol 1987; 24: 221-9.

7. Ismail SM, Colclough AB, Dinnen JS, Eakins D, Evans $\mathrm{DM}$, Gradwell E, et al. Observer variation in histopathological diagnosis and grading of cervical intraepithelial neoplasia. Br Med J 1989; 298: 707-10.

8. Javaheri G, Fejgin MD. Diagnostic value of colposcopy in the investigation of cervical neoplasia. Am J Obstet Gynecol 1980; 137: 588-94.

9. Kirkup W, Hill AS. The accuracy of colposcopically directed biopsy in patients with suspected intraepithelial neoplasia of the cervix. Br J Obstet Gynaecol 1980; 87: 1-4.

10. Ostergard DR, Gondos B. Outpatient therapy of preinvasive cervical neoplasia: selection of patients with the use of colposcopy. Am J Obstet Gynecol 1973; 115: 783-5. 
11.Pearson SE, Whittaker J, Ireland D, Monaghan JM. Invasive cancer of the cervix after laser treatment. Br J Obstet Gynaecol 1989; 96: 486-8.

12.Prendiville W, Cullimore J, Norman S. Large loop excision of the transformation zone (LLETZ). A new method of management for women with cervical intraepithelial neoplasia. Br J Obstet Gynaecol 1989; 96: 1054-60.

13. Reis AFF, Horta ALA, Silveira CMG, Camargo MJ, Dantas N. Valor da citologia e da biopsia orientada pela colposcopia no diagnóstico das neoplasias cervicais uterinas. J Bras Ginecol 1992; 102: 1637.

14.Ronk DA, Jimerson GK, Merrill JA. Evaluation of abnormal cervical cytology. Obstet Gynecol 1977; 49: $581-6$.

15.Sevin BU, Ford JH, Girtanner RD, Hoskins WJ, NG $\mathrm{AB}$, Nordqvist SRB, et al. Invasive cancer of the cervix after cryosurgery. Pitfalls of conservative management. Obstet Gynecol 1979; 53: 465-71.

16.Shingleton HM, Gore H, Austin JM Jr. Outpatient evaluation of patients with atypical Papanicolaou smears: contribution of endocervical curettage. Am J Obstet Gynecol 1976; 126: 122-8.

17.Shumsky AG, Stuart GC, Nation J. Carcinoma of the cervix following conservative management of cervical intraepithelial neoplasia. Gynecol Oncol 1994; 53: 50-4.
18. Silva LFR. Propedêutica da neoplasia intra-epitelial cervical - variante III - uma proposta alternativa. Tese (Mestrado). Rio de Janeiro: Universidade Federal do Rio de Janeiro, 1989.

19.Simões PM, Almeida Filho GL, Vinagre JBF, Meyrelles ARI, Almeida Filho GPA. Estudo comparativo entre colposcopia, citopatologia e histopatologia em 291 pacientes conizadas. Rev Bras Ginecol Obstet $1984 ; 6: 217-22$

20.Souza RC. Conização eletrocirúrgica: apresentação de nova técnica com alça diatérmica. Tese (Doutorado). Rio de Janeiro: Universidade Federal do Rio de Janeiro, 1992.

21.Stafl A, Mattingly RF. Colposcopic diagnosis of cervical neoplasia. Obstet Gynecol 1973; 41: 16876.

22.Townsend DE, Richart RM, Marks E, Nielsen J. Invasive cancer following outpatient evaluation and therapy for cervical disease. Obstet Gynecol 1981; 57: 145-9.

23. Veridiano NP, Delke I, Tancer ML. Accuracy of colposcopically directed biopsy in patients with cervical neoplasia. Obstet Gynecol 1981; 58: 185-7.

24. Wetrich DW. An analysis of the factors involved in the colposcopic evaluation of 2194 patients with abnormal Papanicolaou smears. Am J Obstet Gynecol 1986; 154: 1339-49.

\section{COMUNICADO AOS ASSOCIADOS E LEITORES}

\section{FEBRASCO na Internet}

Para informações e consultas temos à disposição quatro endereços eletrônicos:

$$
\begin{gathered}
\text { febrasgopresidencia@alphanet.com.br } \\
\text { secretaria_executiva@febrasgo.org.br } \\
\text { publicacoes@febrasgo.org.br } \\
\text { tego_habilitacao@febrasgo.org.br }
\end{gathered}
$$

Home page: www.febrasgo.org.br 